\title{
Impacto de las clases virtuales en la educación de estudiantes de Ingeniería Industrial de la Universidad Tecnológica de Panamá durante la pandemia de Covid-19
}

\author{
Impact of virtual classes on the education of Industrial \\ Engineering students of the Technological University of Panama \\ during the Covid-19 pandemic
}

\author{
Renata Rivera ${ }^{I}$, Lya Gutiérrez ${ }^{I}$, Jessica Solis ${ }^{I}$, Rita Araúz- Takakuwa ${ }^{2 *}$ \\ ${ }^{1}$ Licenciatura en Ingeniería Industrial, Facultad de Ingeniería Industrial, Universidad Tecnológica de Panamá, ${ }^{2}$ Departamento de \\ Estadística y Economía, Facultad de Ingeniería Industrial, Universidad Tecnológica de Panamá
}

Resumen En la educación, la crisis sanitaria mundial por el Covid-19 representa la transformación del sistema educativo tradicional presencial a un sistema educativo totalmente virtual, lo cual es un salto tecnológico emergente pero necesario para la continuidad de la preparación profesional. En esta investigación se analizaron los efectos de las clases virtuales en la formación académica de estudiantes, con el fin de dar recomendaciones y sugerencias que aporten al desarrollo de una nueva metodología de enseñanzaaprendizaje virtual capaz de otorgar al estudiante los conocimientos requeridos para su crecimiento académico. Se diseñó una herramienta de medición basada en un cuestionario compuesto por preguntas relacionadas al grado de satisfacción del estudiante con la preparación académica virtual, medida por una escala Likert con la cual se conoce el grado de conformidad del encuestado. Los resultados obtenidos indican que el nivel del aprendizaje virtual se encuentra en un punto regular, donde se considera que la modalidad virtual debe ser diferente a la presencial. Sin embargo, los estudiantes en su mayoría se encuentran moderadamente satisfechos con las clases virtuales. Finalmente, en esta nueva modalidad influyen numerosos factores como el acceso tecnológico de los estudiantes y la metodología del cuerpo docente, entre otros.

Palabras clave Clases virtuales, Covid-19, formación académica, pandemia.

\begin{abstract}
In education, the global health crisis caused by the Covid-19 represents the transformation of the traditional face-to-face educational system to an online educational system, which is an emerging technological leap but necessary for the continuity of professional preparation. This research analyses the effects of virtual classes on students' academic training, in order to provide recommendations and suggestions that contribute to the development of a new virtual teaching-learning methodology capable of providing the student with the knowledge required for academic growth. A measurement tool was designed, based on a questionnaire made up of questions related to the degree of student satisfaction with online classes, measured by a Likert scale in which the degree of satisfaction of the respondent is known. The results indicate that online learning stands at a regular point, on which it is considered that virtual modality should be different from the traditional one. However, most of the students are moderately satisfied with online classes. In addition, this new modality is influenced by numerous factors that contribute to its development, such as the technological access of the students and the methodology of the professor, among others.
\end{abstract}

Keywords Virtual classes, Covid-19, academic training, pandemic.

* Corresponding author: rita.arauz@utp.ac.pa

\section{Introducción}

Debido a la pandemia provocada por el COVID-19 las casas de estudios superiores al no poder recibir a los alumnos en un aula física de clases han tenido que alterar el modelo tradicional de enseñanza- aprendizaje, poniendo en marcha un plan de contingencia basado en la utilización de plataformas

virtuales para solventar los requerimientos académicos de los estudiantes.

La insuficiente planificación previa a la puesta en acción del plan contingente ha generado cierto recelo en la comunidad educativa, debido a la escasez de tiempo con el que contaron las universidades para gestionar el rediseño integral de las 
asignaturas impartidas de forma presencial. Se omitieron aspectos relevantes de influencia en la formación profesional del alumnado como lo son: el nivel socioeconómico de aquellos estudiantes que reciben apoyo de la universidad por medio del comedor universitario, servicios bibliotecarios o conexión gratuita a la red wi-fi de la institución [1]. Se percibe falta de equidad académica al no poder evaluar las condiciones de todos los estudiantes, ignorando a aquellos que no cuentan con los medios tecnológicos mínimos requeridos y el nivel de manejo de las plataformas digitales [2].

\subsection{Ventajas de las TIC's}

El mundo está viviendo la era de las tecnologías de la información y comunicación (TIC`s). Estas han alcanzado a trascender en muchas áreas, en especial el campo educativo [1].

La incorporación de las TIC's en la educación resulta en un nuevo método de enseñanza [2]. Dentro de las ventajas que se manifiestan en este nuevo método se puede encontrar, la presencia, ya que permite al estudiante recibir las clases desde cualquier ubicación y tiempo.

La realidad virtual ha sido estudiada por varios años, se ha reflejado como una modalidad eficaz de formación y evaluación [3]. La misma ha demostrado que puede generar en los estudiantes diferentes beneficios [4], entre ellos podemos destacar la comprensión de contenidos establecidos [3], optimización de creatividad [5], rendimiento de los estudiantes [6], aumento de la participación [7] y el compromiso de los estudiantes [8].

Por consiguiente, ha logrado que la educación sea asequible para aquellos que no la recibían [9], generando que las distintas personas se formen debidamente en aptitudes propias de sus campos de conocimiento [3]. También la implementación de ambientes virtuales para el aprendizaje favorece la inclusión de alumnos y profesores que no puedan desplazarse físicamente hasta el lugar del centro de estudio, siempre y cuando cuenten con los medios electrónicos necesarios.

Los factores que influyen en el desarrollo educativo son: el docente, el estudiante, el contenido o materia y el contexto del aprendizaje.

\subsection{Desventajas de las TIC's}

Un estudio realizado en Florianópolis, Brasil pudo dar a conocer que en los procesos de enseñanza-aprendizaje sobre docentes observados se obtuvo un bajo nivel de aprovechamiento de las TIC's [10]. Estas deben estar enfocadas en la creación del material para el estudiante y no solo como medio de información [11].

Asimismo, un estudio reciente por el Sindicato de Educadores Misioneros Alfredo Bravo [12] ha expuesto que en una clase virtual:

- Se labora con mayor esfuerzo que en una presencial.

- Existen docentes que no tienen conocimientos básicos de las plataformas, incluso del correo electrónico.

- La plataforma puede llegar a venirse abajo.
- No todos cuentan con los medios electrónicos suficientes, algunos solo cuentan con el celular.

\subsection{Proceso de enseñanza-aprendizaje}

Dentro del proceso de enseñanza-aprendizaje es importante destacar el contexto actual de la modalidad virtual. Se deben estudiar las plataformas existentes que satisfagan las necesidades tanto del educador como del estudiante. Estas pueden tener en común que propicien la buena comunicación y llamadas con video para un contacto más cercano, ciertos ejemplos pueden ser, correo Electrónico, WhatsApp, Zoom, Facebook Live, Skype, Microsoft Teams, Discord, entre otras [1].

Existen muchos beneficios al implementar las clases virtuales, sin embargo, para su éxito se debe mantener un equilibrio entre el docente y estudiante. Se deben encontrar las estrategias didácticas y tecnológicas adecuadas para el entendimiento de la información durante el período académico.

En vista que las clases presenciales fueron interrumpidas de una manera inesperada, se ha provocado, especialmente en los centros de estudios superiores, una reingeniería del sistema y por ende el docente ha tenido que iniciar de cero y aprender sobre el transcurso de las clases virtuales.

En estas circunstancias el cuerpo docente ha reestructurado los calendarios y examinado los recursos tecnológicos que impulsen el desarrollo de tácticas adecuadas para el proceso de enseñanza-aprendizaje [13].

\subsection{Estrategias didácticas}

El informe Study of Undergraduate Students and Information Technology (ECAR) 2017 [14] destaca un 75\% de profesores que generalmente utilizan los sistemas tecnológicos institucionales para asignaciones, tales como compartir programa de la materia, recursos bibliográficos, publicar notas, solicitar y recibir las asignaciones. Lo que indica que es necesaria la formación innovadora a los facilitadores para que tengan las habilidades específicas y puedan planear y ejecutar enfocados hacia el estudiante [15].

\subsection{Clases virtuales en Panamá}

La presente investigación busca evaluar si las técnicas andragógicas implementadas a través de las clases virtuales en la educación de los estudiantes de pregrado de la Facultad de Ingeniería Industrial de la Universidad Tecnológica de Panamá satisfacen los requerimientos educativos de los estudiantes.

Una vez obtenidos los resultados, concretaremos la variabilidad que tienen los factores sobre la formación académica de los estudiantes para contribuir a la preparación de la nueva modalidad virtual para la Universidad Tecnológica de Panamá.

\section{Materiales y métodos}

El diseño de esta investigación se desarrolló a partir de un 
estudio en el que participaron estudiantes de pregrado de la Facultad de Ingeniería Industrial de la Universidad Tecnológica de Panamá. La etapa primaria del estudio abarcó la elaboración de un cuestionario cuyos resultados al ser analizados estadísticamente manifestaron tendencias conductuales y académicas que han presentado los estudiantes al recibir clases virtuales. Los análisis estadísticos propuestos abarcan un análisis de las frecuencias de las respuestas por las que optan los estudiantes al desarrollar la encuesta y un análisis de varianza que permitió establecer los factores significativos que inciden en el desempeño conductual y académico de los universitarios. Los datos se sometieron a un análisis de fiabilidad del instrumento de medición para determinar el grado de confiabilidad de las preguntas. Seguidamente se realizó un análisis factorial para determinar la homogeneidad de las preguntas y la existencia de variables latentes que permitieran realizar el análisis de regresión. El análisis de regresión reveló relaciones causales entre las variables latentes definidas como formación académica y clases virtuales.

\subsection{Participantes}

La unidad de análisis de este proyecto investigativo son los estudiantes de la carrera de Ingeniería Industrial correspondientes a la Facultad de Ingeniería Industrial de la Universidad Tecnológica de Panamá. Para realizar el estudio se obtuvo una muestra significativa de la población general de la Facultad por nivel/año de estudio. Ver ecuación 1

$$
\mathrm{n}=\frac{\mathrm{N} \mathrm{Z} Z^{2} \mathrm{pq}}{\mathrm{E}^{2}(\mathrm{~N}-1)+\mathrm{Z}^{2} \mathrm{pq}}=\frac{265 \times 1.645^{2} \times 0.5 \times 0.5}{0.10^{2}(265-1)+1.645^{2} \times 0.5 \times 0.5}=54
$$

En donde:

$\mathrm{n}$ : tamaño de la muestra

$\mathrm{N}$ : población total por nivel de estudio

Z: valor estadístico de una distribución normal para una probabilidad del $90 \%$

p: probabilidad de acierto

q: probabilidad de no acierto

E: error de estimación

\subsection{Hipótesis}

La hipótesis planteada es que la formación académica de los estudiantes de pregrado de la Facultad de Ingeniería Industrial de la Universidad Tecnológica de Panamá es afectada por las clases virtuales.

\subsection{Instrumentos de medición}

La herramienta fue un cuestionario compuesto de preguntas relacionadas al grado de satisfacción que presentan los estudiantes al recibir clases virtuales. A continuación, se definen las variables independientes, dependiente e intervinientes.

- Variable Independiente: clases virtuales; es la nueva modalidad de enseñanza impartida por medio de plataformas digitales.
- Variable Dependiente: formación académica; hace referencia a los diferentes métodos de enseñanza utilizados para impartir conocimiento a un estudiante.

- Variables Intervinientes: tipo de plataforma digital, calidad de la conexión a internet, estado de ánimo de los estudiantes, competencias digitales que poseen los estudiantes, lugar donde se desarrollan las actividades académicas, horario de clases, metodología de enseñanza-aprendizaje de los distintos profesores, entre otras.

\section{Resultados y discusión}

El análisis factorial reveló que la formación académica se define principalmente mediante las siguientes preguntas:

¿La modalidad de enseñanza virtual satisface las necesidades académicas del estudiante?

¿Los docentes explican de manera clara cada tema de estudio?

¿Considera usted que la cantidad de material de estudio facilitado en cada una de las materias virtuales es adecuada?

Para el caso de la variable independiente clases virtuales resultan importantes las siguientes preguntas:

CV1: ¿La plataforma de Microsoft Teams satisface sus necesidades como estudiantes?

CV2: ¿Considera usted que la organización de las clases en modalidad virtual debe ser diferente a la presencial en base a horario, contenido y demás?

CV3: ¿Cumplen los docentes con el horario establecido por la universidad?

CV4: ¿Considera usted que las plataformas virtuales como Zoom y Discord satisfacen sus necesidades como estudiante?

Un aspecto importante como resultado del estudio es que la satisfacción de los estudiantes con la modalidad virtual es regular. Esto se pudo determinar a partir de la pregunta sobre ¿cómo calificaría el nivel de aprendizaje con las clases virtuales?, en la cual se le pide al estudiante responder basado en una escala del 1 al 5, siendo 1 malo, 2 ligeramente malo, 3 regular, 4 bueno y 5 excelente, (ver figura 1). La mayoría de los encuestados considera que el nivel de aprendizaje es regular. Debido a la falta de estudios anteriores sobre el tema, no se tiene información sobre la satisfacción del estudiante con las clases presenciales. El contraste entre las clases presenciales y virtuales representa un tema para futuras investigaciones, ya que debido a limitaciones de tiempo no se pudo ampliar en este estudio.

De los 54 encuestados, 15 se ubican en el nivel 4, indicando un buen grado de satisfacción. 


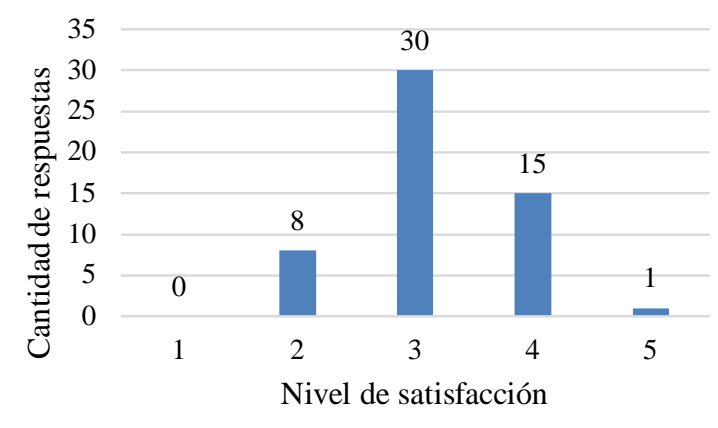

Figura 1. Nivel de satisfacción de los estudiantes sobre la formación académica en la modalidad virtual.

\subsection{Análisis de fiabilidad}

La variable dependiente, formación académica, es fiable con respecto a sus elementos estandarizados debido a que su alfa de Cronbach resultó ser 0.762 , un resultado bueno para una primera encuesta. En el caso de la variable independiente clases virtuales, el alfa de Cronbach resultó en 0.604 lo cual indica un grado aceptable de fiabilidad (ver tabla 1).

Tabla 1. Índice alfa de Cronbach

\begin{tabular}{|c|c|c|c|}
\hline \multicolumn{4}{|c|}{ Estadísticas de fiabilidad } \\
\hline Variable & $\begin{array}{c}\text { Alfa de } \\
\text { Cronbach }\end{array}$ & $\begin{array}{c}\text { Alfa de } \\
\text { Cronbach } \\
\text { basada en } \\
\text { elementos } \\
\text { estandarizados }\end{array}$ & $\begin{array}{c}\text { N de } \\
\text { elementos }\end{array}$ \\
\hline $\begin{array}{c}\text { Formación } \\
\text { académica }\end{array}$ & 0.745 & 0.762 & 10 \\
\hline $\begin{array}{c}\text { Clases } \\
\text { virtuales }\end{array}$ & 0.604 & 0.622 & 10 \\
\hline
\end{tabular}

\subsection{Análisis de regresión}

El análisis de regresión reveló un coeficiente de determinación de 0.2587 , esto indica que $25.87 \%$ de la variabilidad en la formación académica depende de las clases virtuales (ver tabla 2).

Tabla 2. Resultados del análisis de regresión

\begin{tabular}{|c|c|c|c|}
\hline S & R-cuad. & $\begin{array}{c}\text { R-cuad. } \\
\text { (ajustado) }\end{array}$ & $\begin{array}{c}\text { R-cuad. } \\
\text { (pred) }\end{array}$ \\
\hline 0.861013 & $31.46 \%$ & $25.87 \%$ & $13.56 \%$ \\
\hline
\end{tabular}

El análisis contó con un nivel de significancia de $\alpha=10 \%$. Esto nos demuestra que los componentes CV_1, CV_3 y CV_4 son significativamente influyentes en la formación académica debido a que cuentan con un Pvalue menor a su nivel de significancia de acuerdo con lo que se muestra en los resultados de la tabla 3.
Tabla 3. Análisis del modelo estadístico

\begin{tabular}{|c|c|c|c|}
\hline Término & Coeficiente & $\begin{array}{c}\text { Coef. de } \\
\text { estimación }\end{array}$ & Valor P \\
\hline Constante & -0.000 & 0.117 & 1.000 \\
\hline CV_1 & 0.200 & 0.118 & 0.097 \\
\hline CV_2 & -0.061 & 0.118 & 0.607 \\
\hline CV_3 & 0.460 & 0.118 & 0.000 \\
\hline CV_4 & 0.243 & 0.118 & 0.045 \\
\hline
\end{tabular}

Dentro del estudio realizado en estudiantes de pregrado de la Facultad de Ingeniería Industrial, específicamente a estudiantes de cuarto y quinto año, se demuestra que:

- Veintinueve de cada cincuenta y cuatro estudiantes están moderadamente satisfechos con que la modalidad de enseñanza virtual satisface las necesidades académicas del estudiante.

- Treinta y dos de cada cincuenta y cuatro estudiantes están de acuerdo con que los docentes explican de manera clara cada tema de estudio.

- Veinte de cada cincuenta y cuatro estudiantes están de acuerdo con que la cantidad de material de estudio facilitado en cada una de las materias virtuales es adecuada.

- En la figura 1 se observa que treinta de cada cincuenta y cuatro estudiantes dan una calificación regular al nivel de aprendizaje con las clases virtuales.

- Treinta y uno de cada cincuenta y cuatro estudiantes están de acuerdo sobre que los profesores se muestran accesibles al momento del estudiante tener alguna duda o consulta.

\section{Conclusiones}

La educación virtual se puede definir como un método alterno y eficiente para la formación profesional de los estudiantes siempre y cuando se opte por el método de enseñanza-aprendizaje más aceptado por alumnos y profesores.

Con el estudio realizado se puede inferir que una gran parte de la población estudiada se encuentra modernamente satisfecha con la nueva metodología de enseñanza y consideran que este cambio emergente en término general es medianamente aceptable.

Si bien es cierto el cambio a una nueva metodología de enseñanza no es fácil, dada la situación de crisis mundial existente y la emergencia del cambio, se considera que los resultados son aceptables. Sin embargo, actualmente el cambio ya no es desconocido por lo que se pueden implementar nuevos métodos de enseñanza virtual donde la mayoría de los estudiantes se encuentren satisfechos con su formación académica de manera virtual.

Finalmente, una vez concluido el proyecto, se considera interesante realizar otros estudios que aporten al crecimiento de la investigación, se propone: 
Araúz-Takakuwa (et al): Impacto de las clases virtuales en la educación de estudiantes de Ingeniería Industrial de la Universidad Tecnológica de Panamá durante la pandemia de Covid-19

- Ampliar la población objeto de estudio con la finalidad de obtener una muestra variada y representativa que alcance los diferentes niveles académicos que forman parte del tema bajo investigación.

- Analizar los diversos efectos de las clases virtuales en la formación académica desde otras perspectivas como ergonómicas, psicológicas, entre otras.

- Extender los estudios a docentes, estudiantes y herramientas virtuales con el fin de obtener una metodología completa que favorezca la formación académica.

\section{AGRADECIMIENTOS}

Los datos de la población de estudiantes que actualmente estudian la carrera de Ingeniería Industrial en la Facultad de Ingeniería Industrial para este trabajo fueron proporcionados por la Secretaría Académica de la Facultad de Ingeniería Industrial de la Universidad Tecnológica de Panamá.

\section{REFERENCIAS}

[1] K. F. Cáceres-Piñaloza, "Educación virtual: Creando espacios afectivos, de convivencia y aprendizaje en tiempos de COVID19," CienciAmérica, vol. 9, no. 2, p. 38, 2020, doi: 10.33210/ca.v9i2.284

[2] A. V. Blanco, "El rol del docente en la era digital," Rev. Electron. Interuniv. Form. del Profr., vol. 30, no. 2, pp. 103114, 2016

[3] N. Campos Soto, M. Ramos Navas-Parejo, y A. J. Moreno Guerrero, "Realidad virtual y motivación en el contexto educativo: Estudio bibliométrico de los últimos veinte años de Scopus," Alteridad, vol. 15, no. 1, pp. 47-60, 2019, doi: 10.17163/alt.v15n1.2020.04.

[4] A. Klippel et al., "Transforming Earth Science Education Through Immersive Experiences: Delivering on a Long Held Promise," J. Educ. Comput. Res., vol. 57, no. 7, pp. 1745-1771, 2019, doi: 10.1177/0735633119854025.

[5] X. Yang, L. Lin, P.-Y. Cheng, X. Yang, y Y. Ren, "Which EEG feedback works better for creativity performance in immersive virtual reality: The reminder or encouraging feedback?," Comput. Human Behav., vol. 99, pp. 345-351, 2019, doi: https://doi.org/10.1016/j.chb.2019.06.002.
[6] N. E. Cagiltay, E. Ozcelik, M. Berker, y G. G. M. Dalveren, "The Underlying Reasons of the Navigation Control Effect on Performance in a Virtual Reality Endoscopic Surgery Training Simulator," Int. J. Human-Computer Interact., vol. 35, no. 15, pp. 1396-1403, 2019, doi: 10.1080/10447318.2018.1533151.

[7] R. Lorenzo-Alvarez, T. Rudolphi-Solero, M. J. Ruiz-Gomez, y F. Sendra-Portero, "Medical Student Education for Abdominal Radiographs in a 3D Virtual Classroom Versus Traditional Classroom: A Randomized Controlled Trial," Am. J. Roentgenol., vol. 213, no. 3, pp. 644-650, Jul. 2019, doi: 10.2214/AJR.19.21131.

[8] G. Makransky y L. Lilleholt, "A structural equation modeling investigation of the emotional value of immersive virtual reality in education," Educ. Technol. Res. Dev., vol. 66, no. 5, pp. 1141-1164, 2018, doi: 10.1007/s11423-018-9581-2.

[9] S. K. Sood y K. D. Singh, “An Optical-Fog assisted EEG-based virtual reality framework for enhancing E-learning through educational games," Comput. Appl. Eng. Educ., vol. 26, no. 5, pp. 1565-1576, 2018, doi: 10.1002/cae.21965.

[10] E. S. Hung, J. V. Cobos, y A. S. Sartori, "Factores determinantes del aprovechamiento de las TIC en docentes de educación básica en Brasil Un estudio de caso," Perfiles Educ., vol. 38, no. 151, pp. 71-85, 2016.

[11] M. Ferrari, J. Martins y V. Theodoro, "Enseñanza y aprendizaje en las carreras de Relaciones Públicas de Brasil: incorporación de plataformas digitales", Revista Mediterránea de Comunicación: Mediterranean Journal of Communication, vol. 11, no. 2, pp. 311-327, 2020.

[12] A. Borgobello, M. Mandolesi, A. Espinosa, y M. Sartori, "Use of ICT in pedagogical practices of teachers of the Faculty of Psychology of a public university in Argentina," Rev. Psicol., vol. 37, pp. 279-317, 2019, doi: 10.18800/psico.201901.010.

[13] M. Britez, "La educación ante el avance del COVID-19 en Paraguay. Comparativo con países de la Triple Frontera.", 2020. Disponible en: https://preprints.scielo.org/index.php/scielo/preprint/view/22/v ersion/417.

[14] T. Bonacker, J. von Heusinger, y K. Zimmer, Localization in Development Aid: How Global Institutions enter Local Lifeworlds. Routledge, 2016.

[15] S. Guri-Rosenblit, "Distance Education in the Digital Age: Common Misconceptions and Challenging Tasks," Int. J. ELearning Distance Educ., vol. 23, no. 2, pp. 105-122, 2009. 